\title{
PRESENCIA DE CALAHORRA EN LOS TEXTOS FRANCESES DE VIAJES A ESPAÑA DE LOS SIGLOS XVII Y XVIII*
}

\author{
Ignacio Iñarrea Las Heras** \\ Universidad de La Rioja
}

\begin{abstract}
A mi querido compañero Carmelo Cunchillos y a su esposa Carmen, ambos calagurritanos, en testimonio de afecto y gratitud
\end{abstract}

\begin{abstract}
RESUMEN: En este artículo se desea presentar un conjunto de textos franceses sobre viajes a España publicados principalmente en los siglos XVII y XVIII, con el propósito de mostrar cuál es la presencia que Calahorra tiene en ellos. De esta manera, se podrá comprobar qué conocimientos tenían los autores de dichas obras acerca de esta localidad riojana, cuáles eran los aspectos que les interesaron de ella y, en última instancia, cómo la identificaban.

RÉSUMÉ : Dans cet article on veut présenter un ensemble d'œuvres françaises sur des voyages en Espagne publiées principalement pendant les XVII et XVIII ${ }^{e}$ siècles, avec le propos de montrer quelle est la présence de Calahorra dans ces textes. De cette façon, on pourra constater quelles connaissances avaient les auteurs de ces ouvrages sur cette ville de La Rioja (Espagne), quels étaient les aspects qu'ils ont considérés comme les plus intéressants et, en dernière instance, comment ils l'identifiaient.
\end{abstract}

PALABRAS CLAVE: Calahorra, textos franceses, viajes a España, siglos XVII $y$ XVIII.

MOTS CLÉS: Calahorra, textes français, voyages en Espagne, XVII et XVIII siècles.

* Esta publicación está vinculada al proyecto 2009/01 del programa FOMENTA de ayudas a proyectos de investigación, dentro de los Planes Riojanos de I+D+I. Convocatoria 2009. Gobierno de La Rioja. Consejería de Educación, Cultura y Deporte.

** El autor pertenece al Centro de Investigación en Lenguas Aplicadas (CILAP) de la Universidad de La Rioja. 
La ciudad de Calahorra, por su situación en el valle del Ebro, ha sido históricamente lugar de paso para viajeros de todas clases, desde peregrinos hasta fuerzas militares invasoras. Ya desde la Edad Media, gran cantidad de viajeros piadosos procedentes de la Rioja Baja y de la Ribera de Navarra, de Aragón, del Levante, de Cataluña, del sureste de Francia o de Italia se incorporaban en Logroño al Camino Francés, no sin antes haber pasado por otras localidades riojanas como Alfaro, Rincón de Soto o Alcanadre, además de la propia Calahorra, todas ellas enclavadas en el llamado Camino del Ebro ${ }^{1}$. Además, no hay que olvidar acontecimientos como la llegada a esta localidad, en 1366, de las tropas dirigidas por Enrique de Trastámara. Con el apoyo de las Grandes Compañías al mando del francés Bertrand du Guesclin, el hermano bastardo de Pedro I penetró en territorio castellano procedente del reino de Aragón. Y fue precisamente en la ciudad de los Santos Mártires donde fue proclamado rey de Castilla ${ }^{2}$, antes de que se procediese en Burgos a la ceremonia solemne de su coronación.

Así pues, no han faltado extranjeros de diversas procedencias que no solamente conocieron Calahorra, sino que dejaron constancia escrita de su paso por la localidad. Los franceses no fueron, desde luego, una excepción, y ya en los si-

1. Vid. Vázquez de Parga, Lacarra y Uría Ríu (1949, vol. 2: 35-36) y Tobías Tobías, Ochoa Alfaro y Ochoa Moneva (1999: 27-61).

2. La Chronique des règnes de Jean II et de Charles V incluye una breve alusión al paso de Enrique II por Calahorra: “... et aussi que icelui Henri ot sceu que la plus grant partie des gens du dit royaume de Castelle le recevroient volentiers, se il y aloit, il se mist en chemin pour y aler et prist le chemin par les montaignes de Foiez; et jasoit ce que il eust pluseurs empeschemenz, il entra ou dit pays de Castelle, le XXVII jour du mois de septembre de mil CCC LXVII desus dit, et premierement en la cité de Calehorre, et de là ala á Burgues, et fu receu ou dit pays de Castelle de toutes gens moult honnorablement, et luy fist l'en toute obeissance comme à seigneur" (Anónimo, 1910-1920, vol. 2: 36-37). En cambio, Pedro López de Ayala, en su Crónica del Rey Don Pedro, refleja este hecho de forma más detallada: "Desque la cibdad de Calahorra fué asi cobrada, é ovieron nuevas el Conde Don Enrique é los que con él venian como el Rey Don Pedro estaba en Burgos é tenia y sus gentes ayuntadas, é sopieron de cierto que non avia voluntad de pelear con ellos, ovieron alli en Calahorra todos su acuerdo é su consejo. E de todos los estrangeros que alli venian eran los que ordenaban todo el fecho dos, por quanto avian visto muchos fechos de armas y de guerras, de los cuales era el uno Mosen Beltran de Claquin, que era Breton del señorio del Rey de Francia; é el otro Mosen Hugo de Caureley, que era Ingles de Inglaterra. E estos, é los otros estrangeros dixeron al Conde Don Enrique, que pues tan nobles gentes como aquellos que alli venian con él eran acordados de le guardar é tener por Mayor en esta cabalgada, é él avia cobrado una cibdad de Castilla, que le rogaban que se ficiese llamar Rey de Castilla, é tomase título de Rey; ca ellos tenian, segund la snuevas que él sabia de la tierra, que el Rey Don Pedro non daria batalla, nin podia defender el Regno. E en este acuerdo mesmo fueron el Conde de Denia, que fué después Marqués de Villena, é los otros Ricos omes é Caballeros de Aragon que alli venian. E como quier que al Conde Don Enrique luego non le podian traer á esto, pero, segund paresció, plogóle mucho dello. E luego que llegó alli en la dicha cibdad de Calahorra le nombraron Rey, é anduvieron por la cibdad llamando: 'Real, real per el Rey Don Enrique'” (Rosell, ed., 1953, vol. 1: 538). 
glos XVII y XVIII se asiste a la aparición de varios de textos de diversa índole relativos a viajes por España, en los cuales se hace alusión a Calahorra. En el presente trabajo se va a ofrecer una visión de esas producciones escritas, con el propósito de mostrar qué conocimiento tenían de esta ciudad los viajeros procedentes de Francia que pudieron visitarla y mostraron interés por ella.

* $* \quad *$

En 1591 se publicó por primera vez la obra del médico suizo Théodore Turquet de Mayerne (1573-1655) ${ }^{3}$ titulada Sommaire description de la France, Allemagne, Italie \& Espagne. Tuvo un notable éxito, pues fue objeto de al menos catorce reimpresiones hasta $1653^{4}$. A lo largo del siglo XVI, la enemistad entre Francia y España fue un gran obstáculo para la realización de viajes desde el primer país al segundo motivados por el gusto o el interés por conocerlo:

Les affrontements quasi continuels entre les royaumes de France et d'Espagne, avant et pendant les guerres de Religion, avaient créé, il est vrai, des circonstances fort peu favorables à développer en France le goût du voyage en Espagne quand il n'y avait pas nécessité (Bennassar y Bennassar, 1998: III).

Obviamente, las Guerras de Religión tampoco ayudaron al desarrollo de este tipo de iniciativa, aunque fueron igualmente causa de la realización de viajes forzosos fuera de Francia para escapar a la muerte y a la destrucción. Así lo afirma Mayerne en su dedicatoria: "Mais elle [la incõmodité de viajar] est dure à ceux là seuls qui voyagent par necessité, cõme ont fait plusieurs gens de bien ces années passées, contraints par la fureur des guerres ciuiles de laisser leurs naturelles habitations, \& visiter les natiõs estrãgeres" (1618: s. p.).

De todas formas, hubo en esta época franceses que, por diversas razones relacionadas en buena medida con la necesidad, decidieron ir a (e incluso instalarse en) España ${ }^{5}$. Sin duda, Mayerne escribió su obra pensando en viajeros de todo

3. En relación con la vida de Mayerne, vid. Estienne (1936, vol. 1: 17, n. 2).

4. Vid. Estienne (1936, vol. 1: 17-18).

5. "Il y eut cependant bien des raisons pour les sujets du roi de France de passer les Pyrénées: pèlerins de Saint-Jacques, mercenaires en quête d'aventures, de gloire et de butin, commerçants attirés par les bonnes monnaies d'Espagne et les carences économiques du pays qu'il s'agissait de combler, immigrants pauvres si nombreux en Catalogne et Aragon que les 'maris français' y étaient légion, et nombreux encore à Valence, en Castille, voire en Andalousie, pour combler les déficits de main-d'œuvre du pays, tels les ouvriers boulangers, les cuisiniers, les chaudronniers ou les bûcherons; artistes stimulés par la fièvre de bâtir et la multitude de commandes qui procédaient alors des petites cours seigneuriales, des églises, des couvents ou même des villes..." (Bennassar y Bennassar, 1998: II). 
tipo que se dirigiesen no sólo a la Península, sino también a Alemania, Italia o a distintos lugares de la propia Francia. Su propósito fue proporcionarles un texto que contuviese múltiples informaciones sobre dichos territorios y que les sirviese de guía en sus itinerarios ${ }^{6}$. Aproximadamente, las dos terceras partes de este libro están dedicadas a exponer numerosas rutas (con el detalle de sus distintas etapas y la distancia medida en leguas ${ }^{7}$ entre unas y otras) por cada uno de estos cuatro países, así como los caminos de comunicación entre Francia y los otros tres $^{8}$. En lo que respecta a España, tienen aquí un indudable interés los trayectos De Saragosse à Bilbao, De Logrogno à Tudella y De Burgos à Saragosse. En todos ellos aparece Calahorra, además de otras localidades de La Rioja. Así, en el primero de ellos se incluye el siguiente tramo riojano:

$\begin{array}{ll}\text { Alfaro } & 1.4 \\ \text { La Venta } & 1.2 \\ \text { Calorra [Calahorra] } & 1.2 \\ \text { La Venta } & 1.2 \\ \text { Montaguto } & 1 . \text { I } \\ \text { Gonzillo [Agoncillo] } & 1.3 \\ \text { Logrogno V. } & 1.2 \\ \text { (Mayerne, 1618: 242). } & \end{array}$

El segundo presenta un recorrido que discurre casi en su totalidad por La Rioja:

$\begin{array}{ll}\text { Aconciglio [Agoncillo] } & 1.2 \\ \text { La Venta de Lagusceio } & 1.2 \\ \text { La Venta de Seguera } & 1.2 \\ \text { Calaorra v. } & 1.2 \\ \text { La Venta del ricon de Solo [Rincón de Soto] } & 1.2 \\ \text { Alfaro } & 1.2 \\ \text { Tudella } & 1.4 \\ \text { (Mayerne, 1618: 257). } & \end{array}$

6. 'Or quelle qu'en soit la cause tous ceux qui se trouuent en voyage ont un grand soulagement quand ils rencontrent quelque bonne guide, \& leur semble veoir un ange du ciel, lors qu'il se presente homme qui leur peut donner seure addresse de paruenir au lieu où ils pretendent. Ce qui est mis par toutes nations pour un general deuoir de l'hõme raisonnable enuers son prochain, pour lequel le Chrestien doit estimer estre nay autant que pour soy mesme. Ceste consideration [...] a fait que ie me suis mis à dresser une briefue description de l'assiete des quatre principales regions de l'Europe, assauoir de la France, l'Allemagne, l'Italie \& de l'Espagne, \& à faire une particuliere recerche de leurs prouinces, \& des chemins qui conduisent aux lieux plus frequentez \& villes plus renommées d'icelles, $\&$ des foires celebres entre ces nations, à quoy i'ay aussi adiousté un petit traicté de monneyes (Mayerne, 1618: s. p.).

7. Como se podrá ver, tanto aquí como más adelante al hablar de otros trayectos, este dato se indica de forma numérica y con la inicial $l$., que corresponde a la palabra lieue(s) (legua[s]).

8. Vid. Mayerne (1618: 102-269). 
Por último, la tercera ruta es la que da cabida a un mayor número de localidades o lugares situados en territorio riojano:

$\begin{array}{ll}\text { Gragnon } & 1 . \text { I } \\ \text { S. Domingo de la Cakada } & 1 . \text { I } \\ \text { Nagiara } & 1.4 \\ \text { Heremita de S. Anton } & 1 . \text { I } \\ \text { Nauarrette } & 1 . \text { I } \\ \text { Logrogno } & 1.2 \\ \text { Corzinglio [Agoncillo] } & 1.2 \\ \text { Mont Agudo } & 1.3 \\ \text { La Venta } & 1 . \text { I } \\ \text { Calahorra V. } & 1.2 \\ \text { El Aldea } & 1.2 \\ \text { Al Faro } & 1.2 \\ \text { (Mayerne, 1618: 264). } & \end{array}$

Como es sabido, desde principios del siglo XVII la lengua y la literatura españolas gozaban de gran prestigio más allá de los Pirineos, había un gran interés por la cultura hispana. Esta circunstancia podría haber sido un aliciente que animara a muchos franceses a visitar y conocer España. Sin embargo, de modo similar al siglo anterior, los conflictos políticos y bélicos mantenidos entonces entre los reinos francés y español no favorecieron en absoluto la realización de viajes desde Francia a la Península9 . A partir de mediados de siglo, la situación, en este aspecto, empieza a cambiar. Como señalan Bennassar y Bennassar, se podría pensar que

les victoires françaises sur les fameux tercios espagnols à Rocroi (1643), puis à Lens (1648) libérèrent l'opinion française de la peur de l'Espagne qui avait dominé jusque-là. Dans ces conditions [...], l'Espagne reçoit alors des voyageurs français soucieux de découvrir le pays et d'en proposer une image, non dénuée de préjugés, il va sans dire, à leurs concitoyens. Plusieurs d'entre eux, ainsi Antoine de Brunel ou François Bertaut, parcoururent le pays du nord au sud ... (1998: V).

Efectivamente, en la segunda mitad del siglo XVII se asiste a la llegada a territorio español de no pocos viajeros y escritores procedentes de Francia, entre los que cabe mencionar, además de los dos anteriores, a Jacques Carel de Sainte Garde, al abad Muret, al abad de Montreuil, al marqués de Villars, al duque de Saint-Simon, a Madame de Gudannes, a su hija la condesa de Aulnoye o a François de Tours $^{10}$.

9. Vid. Bennassar y Bennassar (1998: III-V).

10. Vid. Bennassar y Bennassar (1998: V-VI). 
El más interesante de todos ellos, para los fines del presente trabajo, es François Bertaut, consejero en el Parlamento de Ruán y prior del Monte de los Enfermos. En 1659 hubo de acompañar al mariscal De Grammont, cuando éste fue enviado con el encargo de pedir la mano de María Teresa de Austria para Luis XIV. Tal matrimonio había sido acordado en la Paz de los Pirineos, firmada en $1657^{11}$. La madre de Bertaut era española y él conocía bien el castellano ${ }^{12}$. Cuando el mariscal hubo cumplido la misión encomendada, volvió a Francia. Sin embargo, Bertaut

décida, avec quatre autres Français, "qui voulurent bien faire le voyage d'Andalousie", de poursuivre l'aventure. Il accomplit un long périple à travers la Péninsule [...], de telle sorte que sa vision de l'Espagne est beaucoup plus ample que celle de Brunel, dont le voyage est presque contemporain” (Bennassar y Bennassar, 1998: 1203).

A Bertaut se deben dos obras sobre España: Relation d'un voyage d'Espagne où est exactement décrit l'estat de la Cour de ce royaume et de son gouvernement (1664) y Journal du voyage d'Espagne (1669) ${ }^{13}$. En el transcurso de su viaje tuvo que atravesar La Rioja, procedente de Navarra y en dirección a Castilla. Pasó por las localidades de Calahorra, Logroño, Nájera y Santo Domingo. Sobre la primera de estas ciudades hace los siguientes comentarios:

\begin{abstract}
A l'égard de Calahorre, c'est une vilaine ville assez grande, mais qui paroist par les ruines que l'on voit, avoir esté encore bien plus grande. C'est l'ancienne Calaguriis, d'où quelques-uns croyent que Quintilien estoit natif, que l'Empereur Galba amena avec luy à Rome; car pour Martial il estoit de Calatajut. Celuy qui en est Evesque, l'est aussi de Santo Domingo de la Calçada, de saint Dominique de la chaussée: \& ces deux Eveschez joints ensemble, valent environ vingt mille ducats de rente, ce qui n'est pas beaucoup en Espagne, où tous les Eveschez sont pour l'ordinaire plus estendus \& de plus grand revenu qu'en France. [...]

Calahorre est presentement de Castille, quoy qu'anciennement elle fust de Navarre, mais depuis l'union des Royaumes, on borne celuy de Navarre à l'Ebre. Les Calaguritains du temps des Romains estoient reputez braves soldats (Bertaut, 1919: 16-17).
\end{abstract}

Estas últimas palabras sobre el valor de los guerreros calagurritanos hacen referencia a que, en tiempos de la dominación romana, su fama de valerosos fue tan considerable que Octavio Augusto decidió crear una guardia personal con soldados de esta localidad.

11. Vid. Bennassar y Bennassar (1998: 1203) y García Mercadal (1999, vol. 3: 391).

12. Vid. Bennassar y Bennassar (1998: 1203).

13. Vid. Bertaut (1919). 
A finales del siglo XVII, las peregrinaciones francesas no habían desaparecido, ni mucho menos. Una buena muestra de ello, aunque sea en un sentido negativo, son las ordenanzas, edictos y declaraciones promulgadas por Luis XIV en 1655,1671 y 1686 , para establecer un necesario control en la actividad de peregrinación con destino al extranjero ${ }^{14}$. Para poder practicarla, se hizo obligatorio obtener una autorización oficial por escrito ${ }^{15}$. El motivo de la adopción de tales medidas fue la proliferación de falsos romeros que no eran más que vagabundos, vagos, pícaros o delincuentes disfrazados con los atributos propios de esta clase de viajeros (bordón, sombrero de ala ancha, esclavina). Si existía la falsa peregrinación, era precisamente porque la peregrinación auténtica seguía practicándose y ambas podían confundirse, en beneficio de tales farsantes.

Otro testimonio de esta realidad es la existencia de textos como el libro de la cofradía de peregrinos de la localidad de Senlis (Francia), comenzado en 1680, en el cual se recogen varios itinerarios de peregrinación a Compostela. Cada uno de ellos reproduce (indicando también sus etapas y la distancia en leguas entre ellas) algún tramo de las rutas hacia Compostela, San Salvador de Oviedo o Montserrat. El recorrido que presenta aquí mayor interés es el comprendido entre Santo Domingo de la Calzada y la localidad francesa de Toulouse. Se trata de un camino de peregrinación y, al mismo tiempo, de regreso a Francia. Por ello, aparece con el título Retour de Saint-Dominique par Saragosse, Mont-Serrat, etc ${ }^{16}$. Los dos santuarios españoles más importantes mencionados en el mismo son la basílica de Nuestra Señora del Pilar, en Zaragoza, y el monasterio de Montserrat. Las etapas iniciales de este itinerario (coincidente en parte con la ruta jacobea del Ebro) son poblaciones riojanas y entre ellas se encuentra Calahorra:

Sy le pellerin veut faire le voyage de Nostre-Dame de Mont-Sarrat, il peut prendre le chemin qui s'ensuit:

14. Sobre este asunto tambien se promulgó una ordenanza en 1717 y una declaración real en 1738. Vid. FERPEL (2009).

15. La ordenanza de 1655 establecía al respecto lo siguiente: "Sa Majesté ayant receu diverses plaintes de la part des Bourgeois et Habitans de plusieurs Villes ou Bourgs de ce Royaume, de ce que leurs enfans, soubs prétexte d'aller en Pellerinage à Saint-Jacques de Galice, ou ailleurs hors de ce dit Royaume, se debauchent, quittent leurs maisons, et s'accostent souvent de meschantes compagnies pour faire ces Pellerinages. [...] Sa majesté a défendu et défend très expressément à toute personne de quelque qualité et condition qu'elles soient d'aller en Pellerinage hors du Royaume, sans Passe-port expres de sa Majesté, lequel ne sera expédié à ceux qui voudront faire ces Pellerinages que sur le consentement que leurs père et mère (ou en cas de déceds d'eux, de leurs plus proches parents) auront presté par devant le Juge Royal du lieu de leur demeure, ou du plus prochain, et dont ils raporteront acte authentique" (FERPEL, 2009).

16. Vid. Müller (1914: 191-193). 
De Saint-Dominique à Grenoble [Grânon] ${ }^{17} \quad 31$.

Navarin [Najera] 31.

Caroles [Calahorra] 31.

La faire [Alfare] 31.

(Müller, 1914: 191).

Este libro recoge también una canción cuyo título es Chanson nouvelle sur tous les passages et lieux remarquables qu'il y a aux chemins de Saint-Jacques, Saint-Salvateur et Mont-Sarra. Formaría parte del conjunto de composiciones que cabría identificar como canciones populares francesas de peregrinación jacobea, que proliferaron a lo largo de la Edad Moderna. De ellas existen varios tipos, y la que aquí nos ocupa pertenece al grupo de las canciones de itinerario o cantos-guía. Su función principal era proporcionar a los peregrinos orientaciones e informaciones de considerable variedad, muy útiles para la realización de su viaje a Compostela (descripción del itinerario en sus principales etapas, hospitales y albergues, otros santuarios dignos de ser visitados durante el camino, etc.). La Chanson nouvelle... describe el recorrido desde Senlis, situada al norte de París, hasta Santiago de Compostela. También da cabida al paso por Asturias. El trayecto de regreso a Francia se presenta, al igual que el camino anterior, como una ruta de peregrinación al monasterio de Montserrat: después de atravesar las regiones de Galicia, León y Castilla, las tierras riojanas aparecen como el inicio de un desvío con respecto al Camino Francés que ha de llevar hasta Cataluña y que también se identificaría con un tramo del Camino del Ebro recorrido en sentido inverso. Calahorra es una de la etapas que forman parte de este trayecto:

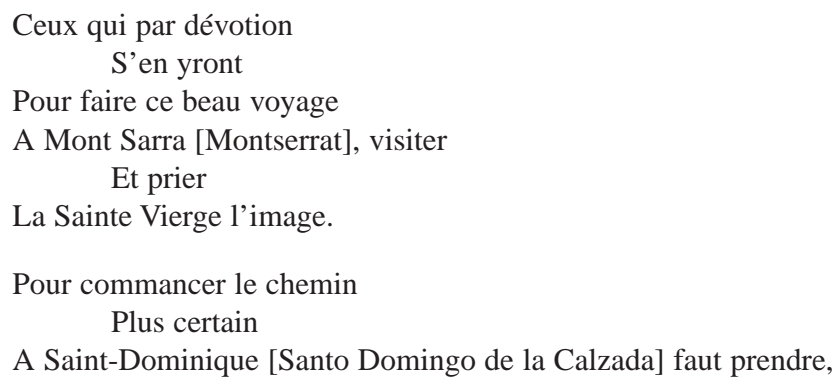

17. Las aclaraciones entre corchetes que se ven en esta cita han sido obra de Müller. Su ortografía no es del todo correcta, ya que Grânon se debe escribir Grañón, Nájera lleva acento en la primera $a$ y Alfare es Alfaro. Grenoble es, efectivamente, Grañón. Sin embargo, aparece situado por error entre Santo Domingo de la Calzada y Navarin. Por último, Navarin no es Nájera, sino Navarrete. Vid. Iñarrea Las Heras (2004-2005: 135-137). 
Passer de là à Grenoble ${ }^{18}$

Ou Carolle [Calahorra]

Pour à Tudelle [Tudela] se rendre

(Müller, 1914: 210. vv. 235-246).

En el siglo XVIII aparecen viajeros y escritores franceses que se interesarán por España, desde una perspectiva influida por la corriente de la Ilustración. Llegarán a adquirir un conocimiento amplio y profundo sobre el país. Esto será determinante en su censura de aquellos aspectos de este reino que consideren especialmente negativos, reflejo de su atraso en todos los sentidos ${ }^{19}$. Sin embargo, tampoco faltará entre ellos una actitud que, sin renunciar a un espíritu crítico y racional (la razón es el arma fundamental para favorecer el conocimiento y el progreso del ser humano), estará caracterizada igualmente por la honestidad y la mesura en los juicios y apreciaciones realizados en relación con dicho territorio:

... Jean François Peyron, Jean-François Bourgoing ou Alexandre de Laborde (qui publiera au début du XIX ${ }^{\mathrm{e}}$ siècle) se montrent modérés, très nuancés dans leurs jugements ou diagnostics; or, ces trois hommes ont parcouru la plus grande partie du pays dont ils ont une expérience approfondie [...]. Pour la première fois, depuis Brunel ou Bertaut au milieu du XVII ${ }^{\mathrm{e}}$ siècle, le lecteur se trouve en présence de voyageurs qui ont voulu connaître et comprendre le pays visité, qui ont consacré des années à cette entreprise et qui ont livré au public une vision sincère, sans passion, de l'Espagne, même si on peut les juger trop "raisonnables", trop soucieux d'apprécier le pays à l'aune des progrès de la raison et de le mettre en chiffres (Bennassar y Bennassar, 1998: VI-VII).

A las aportaciones de estos tres autores hay que unir las de otros textos, en los cuales también puede apreciarse la presencia del espíritu ilustrado. Todos ellos muestran un detallado conocimiento de la compleja realidad española de la época, demostrable precisamente en el hecho de que den cabida a la mención de ciudades de pequeño tamaño y de importancia que cabría calificar como de relativamente menor, tales como la que aquí nos ocupa (o también Tudela, Soria, Calatayud, Segovia, Ávila, Badajoz, Tarragona, etc). Hacia 1729 apareció la obra anónima Lettres ecrites de Madrid en MDCCXXVII sur l'Estat present de la Monarchie

18. Grañón aparece, también aquí, ubicado de forma equivocada. Ha sido colocado entre Santo Domingo de la Calzada y Calahorra. La existencia de este error tanto en el itinerario como en la canción ayudaría a demostrar que el desconocido autor de la Chanson nouvelle... pudo servirse de los trayectos del libro de la cofradía de peregrinos de Senlis en su labor de composición. Vid. Iñarrea Las Heras (20042005: 135-137).

19. "Les philosophes français des Lumières ont fort maltraité l'Espagne: fanatisme, intolérance, obscurantisme, despotisme... L'Inquisition, évidemment vilipendée, devient l'institution emblématique d'un pays décadent" (Bennassar y Bennassar, 1998: VI). 
d'Espagne, où l'on y décrit en Sections les quatorze Royaumes ou Provinces qui la composent. Se presenta como una colección de ocho cartas en las que, de forma bastante minuciosa, objetiva y desapasionada se describen distintos aspectos de España, como el carácter de sus habitantes, la forma de vestirse y peinarse de las damas de la corte, la organización de la Iglesia o el Ejército. La octava carta se divide en catorce partes o secciones, dedicadas a describir, como anticipa el título, los cartorce territorios que componen el reino español. En las páginas en las que se trata sobre Castilla la Vieja (sección 11) hay un pasaje sobre Calahorra, que es una de las sedes episcopales de esta región:

LA VILLE DE CALAHORA. Calahora, en Latin, Calagurris, a le Titre d'Evêché, suffragant autrefois de Tarragone, \& aujourd'hui de Burgos: cette Ville est située sur l'Ebre, qui y reçoit la Riviere de Cidaers de Castiglia [Cidacos]. L'Evêché de la Calzada fut uni à celui de Calahora en 1236. Quintilien, \& Prudence étoient natifs de Calahora (Anónimo, [1729]: 95).

El texto anónimo titulado État politique, historique \& moral du Royaume d'Espagne l'an MDCCLXV constituye un detallado estudio acerca de la España de mediados del siglo XVIII ${ }^{20}$. Aborda facetas numerosas y variadas, tales como la geografía y la historia de este país, sus instituciones, sus costumbres, su situación política, etc. Es un texto en el que tampoco falta la crítica de aquello que su desconocido autor ve como negativo o perjudicial para España. Esto podría llevar, según dice él en la introducción de su obra, a malinterpretar sus verdaderas, y constructivas, intenciones:

La malice Humaine cherchera à y [en este texto] reconnoitre ses traits, et la pluspart des Lecteurs s'arrêteront à l'Ecorce sans aller jusqu'à la Substance. On méconnoitra le but de mon ouvrage, si on n'y cherche que la satire, ou même la critique.

Je me suis fait un Plan d'utilité pour la Nation Espagnole: mais comme la vérité a seule guidé ma Plume, a dicté toutes mes Réflexions, a tracé tous mes tableaux, je crains que l'effet ne réponde pas à mes vues. Plus d'une personne montrera du ressentiment, parce qu'effectivement plus d'une personne est attaquée. L'Inquisition acharnée sonnera l'allarme sur la Religion ${ }^{21}$. Cette Levée de Bouclier suffit aux yeux

20. Vid. al respecto Tamarit Vallés (2003: 113-117 y 473-477).

21. Efectivamente, la Inquisición recibe duros ataques por parte del anómino autor de esta obra: "Le Tribunal de l'Inquisition, ce funeste monument de la Barbarie et du Fanatisme, est le frein des Espagnols et même de leurs souverains. Ce tribunal indépendant de tout pouvoir humanin, emprisonne, juge, fait disparoitre qui luy plait sans que personne puisse murmurer. [...] A present que ce Tribunal n'a plus d'ennemis particuliers, il attaque toute la societé. Les Rois, les grands, personne n'est à l'abri de ses Coups, pas même les Etrangers. On dit à la vérité qu'il est beaucoup plus doux et plus sage qu'autrefois; il ne fait plus bruler pieusement des malheureux en triomphe: mais c'est toujours le siege de la tirannie, et ce qu'il y a de plus révoltant c'est que c'est sur les Etrangers particulierement qu'il sêvit le plus" (Anónimo, 1963: 470-472). 
de la crédule ignorance, pour convaincre indubitablement que j'ay profané le Sanctuaire, pour faire bruler le Livre, ou au moins le suprimer (Anónimo, 1963: 377).

El creador de esta obra señala que su propósito fundamental, con la publicación de la misma, "c'est de dire aux Espagnols des verités dont ils puissent faire leur Profit" (Anónimo, 1963: 379).

En este État politique, historique \& moral du Royaume d'Espagne l'an MDCCLXV se dedican en su primer capítulo (acerca de la geografía de España) unas palabras a la historia de Calahorra. En la descripción de Castilla la Vieja, se dice:

Burgos, la Capitale [de Castilla la Vieja], est une grande ville fort peuplée, connue par un beau crucifix [...]: elle tient le premier rang dans les Etats de Castille. Au Nord, sur les Frontieres de la Navarre, on trouve les villes de Logrogno et Calahorra; la derniere est celebre par sa fidelité à ses souverains, et par la naissance du fameux Rheteur Quintilien, le maitre de la critique, qui a fait de bien mauvais disciples (Anónimo, 1963: 405).

El comentario acerca de la fidelidad de Calahorra a sus soberanos se basa seguramente en que, durante las Guerras Sertorianas (83 a. C.-72 a. C.), esta ciudad se mantuvo fiel de forma inquebrantable a Sertorio.

Joseph de La Porte (1714-1779), el abad de Fontenai (1736-1806) y Louis Domairon (1745-1807) fueron los compiladores de Le voyageur françois, ou La connoissance de l'ancien et du nouveau monde, vastísima colección de relatos de viajes presentados (al igual que Lettres ecrites de Madrid...) en forma de cartas. Fue publicada en 42 volúmenes entre 1765 y 1795. Al supuesto autor se le identifica simplemente como el Voyageur François. El volumen 16 está dedicado a España. El espíritu ilustrado con el que se exponen aquí también (aunque de forma no muy ordenada) los más diversos aspectos acerca de este reino (historia, economía, cultura, etc.) se anuncia ya en el volumen 1: la introducción general de la publicación establece el objetivo fundamental de la obra, claramente didáctico:

En portant, dans ses voyages, le flambeau de la philosophie \& de l'observation, il [el Voyageur François] y puise des connoissances utiles, qu'il communique à ses concitoyens. Tous les objets faits pour exciter la curiosité d'un lecteur philosophe, les loix, les mœurs, les usages, la religion, le gouvernement, le commerce, les sciences, les arts, les modes, l'habillement, les productions naturelles, en un mot, la connoissance de tous les pays \& de toutes les nations de l'univers, en commençant par les peuples de l'Asie, font la matière de toutes ses Lettres. Il ne porte son attention que sur ce qui lui paroît mériter une juste curiosité; \& comme son but est d'intéresser \& d'instruire, tout ce qui ne produit point ces deux effets, ne lui semble pas digne de ses remarques (La Porte, Fontenai y Domairon, 1765-1795, vol. 1: vii-viii). 
Dicho espíritu queda confirmado, por ejemplo, en apreciaciones sobre la Inquisición $^{22}$ (al igual que en el texto inmediatamente anterior) o acerca del proyecto de repoblar Sierra Morena ${ }^{23}$. En relación con Calahorra, se incluyen algunas informaciones de tipo histórico y literario:

Calahorra s'est rendue recommandable par son ancienne fidélité à ses souverains. Auguste voulut avoir, parmi ses gardes, un bataillon de soldats pris dans le nombre de ses habitans. Un autre avantage dont elle se fait gloire, est d'avoir vu naitre le fameux rétheur Quintilien. Tout le monde n'est cependant pas d'accord sur cet article; car plusieurs croient qu'il est né à Rome. On sait qu'il y enseigna publiquement la rhétorique, \& qu'il y composa ces fameuses institutions oratoires, que l'abbé Gédoyn a traduites en notre langue. Elles demeurerent inconnues jusqu'au quinzieme siecle, que le Pogge les trouva, dit-on, dans une vieille tour de l'abbaye de saint Gal (La Porte, Fontenai y Domairon, 1765-1795, vol. 16: 338).

Los textos vistos aquí permiten comprobar que los distintos tipos de viajeros franceses que visitaron Calahorra a lo largo de los siglos XVII y XVIII vienen a coincidir, al menos en gran medida, en resaltar los siguientes aspectos de Calahorra, como rasgos que para ellos definen la identidad de esta ciudad:

- La importancia de su pasado histórico, sobre todo en lo que respecta a la Antigüedad: se destaca aquí su condición de ciudad romana, palpable en sus ruinas; el hecho de ser el lugar de nacimiento del retórico Marco Fabio Quinti-

22. La actitud del autor ante la Inquisición es indudablemente crítica, si bien considera que esta institución ha perdido en el siglo XVIII parte de su poder (como ha quedado indicado en la cita de la nota anterior): "Tel est Madame, ou plutôt tel a été pendant long-tems ce tribunal destructeur, problême étonnant pour toutes les nations, objet d'horreur pour les unes, de vénération pour les autres, \& qu'on est toujours surpris de voir subsister, parmi les peuples policés, dans le sein même du christianisme. Je sais qu'on lui impute des atrocités qu'il ne commet point; mais pourquoi chercher dans le mensonge de quoi le rendre odieux? La vérité seule ne suffit-elle pas pour le faire détester? J'avoue pourtant, que dans ce siecle humain, poli \& éclairé, l'Espagne \& le Portugal ont sagement tempéré la rigueur de ses maximes, \& diminué l'excès de son pouvoir. Les inquisiteurs même sont devenus plus circonspects, moins inflexibles \& plus équitables" (La Porte, Fontenai y Domairon, 1765-1795, vol. 16: 71).

23. La visión de este proyecto está marcada por un claro escepticismo por parte del autor: "Le projet de la peupler [Sierra Morena] fut proposé à la cour en 1725; mais on y trouva alors des difficultés insurmontables. [...] Ce même projet vient d'être présenté de nouveau; mais, s'il est accepté, je doute qu'il puisse jamais être exécuté. On parle de faire venir des étrangers, \& principalement des Allemands \& des Flamands, pour les établir dans ces montagnes... [...] En supposant même qu'aucune des mesures à prendre pour le succès de la colonie ne puisse manquer [...], on peut encore assurer que l'opération sera sans effet, \& que la colonie diminuera d'elle-même; parce que la dépopulation de l'Espagne a sa cause dans le manque de travail, de commerce \& d'industrie; \& que le zele des colons s'éteindra comme celui des anciens habitans" (La Porte, Fontenai y Domairon, 1765-1795, vol. 16: 464-465). 
liano (39-95) y del poeta latino Aurelio Prudencio (348-410); la bravura de los soldados procedentes de allí, en tiempos del imperio romano; la gran lealtad a sus señores, probablemente en referencia a que en todo momento defendió su postura favorable a Sertorio en el transcurso de las Guerras Sertorianas.

Sin embargo, Bertaut no olvida la etapa medieval de su historia, y por ello hace referencia a su anterior pertenencia al reino de Navarra.

- Su condición de sede episcopal, que le confiere una cierta importancia dentro del territorio de Castilla la Vieja.

- Su situación geográfica en el valle del Ebro, zona limítrofe de Castilla la Vieja.

Por otra parte, del siglo XVII nos llegan también algunos textos con breves (pero en absoluto desdeñables) indicaciones sobre Calahorra:

- Las alusiones a esta ciudad como etapa de peregrinación ubicada en el Camino del Ebro. Éste es otro elemento identificador de esta localidad de innegable interés. Bien es verdad que los dos textos citados aquí no presentan esta ruta como un camino en dirección a Compostela, sino como vía de regreso a Francia, previo paso por Zaragoza y Montserrat. De todas formas, sí dejan claro que quienes la transitan son peregrinos franceses que han ido a Compostela y es evidente que, tomada en sentido inverso, lleva a conectar, como se ha dicho, con el Camino Francés. Por lo tanto, la dimensión jacobea de Calahorra es otro elemento que, de una forma u otra, no deja de estar presente en la canción y en el itinerario de peregrinación mencionados.

Así pues las obras sobre viajes franceses en el período indicado demuestran con claridad el estimable conocimiento de sus autores sobre diversos aspectos de Calahorra, en especial su situación, su historia y su cultura.

\section{Bibliografía}

ANÓNIMO (1729): Lettres écrites de Madrid en MDCCXXVII sur l'Estat present de la Monarchie d'Espagne, où l'on y décrit en Section les quatorze Royaumes ou Provinces qui la composent, Béziers: Estienne Barbut. [Versión en línea disponible en http://gallica.bnf.fr (Bibliothèque nationale de France)].

ANÓNIMO (1910-1920): Chronique des règnes de Jean II et de Charles V, 4 tomos en tres volúmenes, ed. R. Delachenal, París: H. Laurens. [Versión en línea disponible en http://gallica.bnf.fr (Bibliothèque nationale de France)].

ANÓNIMO (1963): "État politique, historique \& moral du Royaume d'Espagne l'an MDCCLXV", Revue Hispanique, 30, pp. 376-514. Reimpresión de la edición de 1914. 
BENNASSAR, Bartolomé y BENNASSAR, Lucille (1998): Le Voyage en Espag$n e$. Anthologie des voyageurs français et francophones du XVI ${ }^{e}$ au XIX siècle, París: Robert Laffont.

BERTAUT, François (1919): “Journal du voyage d'Espagne (1659)", Revue Hispanique, 47, 111, pp. 1-317.

ESTIENNE, Charles (1936): La Guide des chemins de France de 1553 par Charles Estienne, 2 vols., ed. Jean Bonnerot. París: Honoré Champion.

FERPEL (2009): "Les réglementations des pèlerinages à l'étranger sous Louis XIV (1643-1715) et Louis XV (1715-1774)" in SaintJacquesInfo. Les textes qui ont fait Compostelle. [Versión en línea disponible en http://lodel.demo.inist.fr/saintjacquesinfo/index.php?id=75].

GARCÍA MERCADAL, J. (1999): Viajes de extranjeros por España y Portugal. Desde los tiempos más remotos hasta comienzos del siglo XX, 6 vols., [Valladolid]: Junta de Castilla y León, Consejería de Educación y Cultura.

IÑARREA LAS HERAS, I. (2004-2005): "Presencia de La Rioja jacobea en la literatura francesa", Epos. Revista de Filología, 20-21, pp. 117-141.

LA PORTE, Joseph de FONTENAI, abad y DOMAIRON, Louis (1765-1795): Le voyageur françois, ou La connoissance de l'ancien et du nouveau monde, 42 vols., París: Vincent, Moutard, Cellot. [Versión en línea disponible en http://gallica. bnf.fr (Bibliothèque nationale de France)].

MAYERNE, Théodore Turquet de (1618): Sommaire description de la France, Allemagne, Italie \& Espagne, avec la gvide des chemins pour aller \& venir par les prouinces, \& aux villes plus renommées de ces quatre regions, [s. 1.]: Iacob Stœr. [Versión en línea disponible en http://gallica.bnf.fr/ark:/12148/bpt6k106 526b.r=theodore+turquet.langES].

MÜLLER, E. (1914): "Une confrérie de Saint-Jacques à Senlis", Bulletin de la Société Historique de Compiègne, 16, pp. 161-222.

ROSELL, C (ed.) (1953): Crónicas de los reyes de Castilla desde don Alfonso X el Sabio hasta los Católicos don Fernando y doña Isabel, 3 vols., Madrid: Atlas (Biblioteca de Autores Españoles, 66, 68, 70).

TAMARIT VALLÉS, I. (2003): Representaciones de la mujer española en el imaginario francés del siglo XVIII, Valencia: Universitat de Valencia, Servei de Publicacions. [Versión en línea disponible en http://www.tesisenxarxa.net/ TDX/TDX_UV/TESIS/AVAILABLE/TDX-0713104-142249/tamarif.pdf].

TOBÍAS TOBÍAS L., OCHOA ALFARO, Á.J. y OCHOA MONEVA, J.C. (1999): Calahorra: en ruta hacia Santiago, Logroño: Gobierno de La Rioja, Instituto de Estudios Riojanos.

VÁZQUEZ DE PARGA, L., LACARRA, J.Mª y URÍA RÍU, J. (1949): Las peregrinaciones a Santiago de Compostela, 3 vols., Madrid: Consejo Superior de Investigaciones Científicas. 